\title{
Huntingtin-associated protein 1 interacts with Ahi1 to regulate cerebellar and brainstem development in mice
}

\author{
Guoqing Sheng, ${ }^{1}$ Xingshun Xu, ${ }^{1}$ Yung-Feng Lin, ${ }^{1}$ Chuan-En Wang, ${ }^{1}$ Juan Rong, ${ }^{1}$ Dongmei Cheng, ${ }^{1}$ \\ Junmin Peng, ${ }^{1}$ Xiaoyan Jiang, ${ }^{2}$ Shi-Hua Li, ${ }^{1}$ and Xiao-Jiang Li ${ }^{1}$ \\ ${ }^{1}$ Department of Human Genetics, Emory University School of Medicine, Atlanta, Georgia, USA. ${ }^{2}$ Terry Fox Laboratory, British Columbia Cancer Agency and \\ Department of Medical Genetics, University of British Columbia, Vancouver, British Columbia, Canada.
}

\begin{abstract}
Joubert syndrome is an autosomal recessive disorder characterized by congenital malformation of the cerebellum and brainstem, with abnormal decussation in the brain. Mutations in the Abelson helper integration site 1 gene, which encodes the protein AHI1, have been shown to cause Joubert syndrome. In this study, we found that mouse Ahi1 formed a stable complex with huntingtin-associated protein 1 (Hap1), which is critical for neonatal development and involved in intracellular trafficking. Hap1-knockout mice showed significantly reduced Ahi1 levels, defective cerebellar development, and abnormal axonal decussation. Suppression of Ahi1 also decreased the level of Hap1; and truncated Ahi1, which corresponds to the mutations in Joubert syndrome, inhibited neurite outgrowth in neuronal culture. Reducing Hap1 expression suppressed the level and internalization of TrkB, a neurotrophic factor receptor that mediates neurogenesis and neuronal differentiation, which led to decreased TrkB signaling. These findings provide insight into the pathogenesis of Joubert syndrome and demonstrate the critical role of the Ahi1-Hap1 complex in early brain development.
\end{abstract}

\section{Introduction}

Clinical features of Joubert syndrome (JS) include neonatal hypotonia (loss of muscle tone), ataxia, developmental delay, mental retardation, and frequently abnormalities in breathing and eye movements (1-3). Various abnormalities affecting a number of systems in the midbrain and hindbrain have been identified as common to JS. JS is characterized by the absence or incomplete formation of the cerebellar vermis, a brain structure thought to be important for control of balance, regulation of muscle tone, and rapid eye movements. The absence of decussation of the superior cerebellar peduncles as well as the corticospinal tracts at the medullary pyramids suggests that JS patients may have a defect in axon guidance and altered brain connections. Defective neuronal proliferation is likely to account for the diminished density of neurons in the cerebellar granule layer, the dentate nuclei of the cerebellum, and the brainstem (1-3).

Forms of JS have been mapped to 3 genetic loci: 9q34.3 (JBTS1: OMIM 213300), 11p12-q13.3 (JBTS2: OMIM 608091), and 6q23 (JBTS3: 608629) (4-6). The developmental defects in cerebellar structures in JS led to the investigation of candidate genes that are important for early development of the cerebellum. However, no mutations in any genes known to contribute to cerebellar development have been identified (7-9). Instead, JBTS3 was found to associate with mutations in the Abelson helper integration site 1 gene $(A H I 1)(10,11)$. Mouse Abi1 was initially identified as a common helper provirus integration site for murine leukemias and lymphomas (12). The protein encoded by the human AHI1 gene

Nonstandard abbreviations used: Ahi1, Abelson helper integration site 1; BDNF, brain-derived neurotrophic factor; GST, glutathione-S-transferase; Hap1, huntingtinassociated protein 1; JS, Joubert syndrome; NGF, nerve growth factor; TrkB, tyrosine kinase receptor $\mathrm{B}$.

Conflict of interest: The authors have declared that no conflict of interest exists. Citation for this article: J. Clin. Invest. 118:2785-2795 (2008). doi:10.1172/JCI35339.
(AHI1 or Jouberin) contains 7 WD40 repeats, an SH3 domain, potential SH3 binding sites, and an $\mathrm{N}$-terminal coiled-coiled domain (13). WD40 domains are present in proteins that are involved in a variety of functions, including signal transduction, RNA processing, transcriptional regulation, cytoskeleton assembly, vesicle trafficking, and cell division (14). SH3 domains are a common feature of signaling molecules involved in numerous pathways (15). Most mutations of the AHI1 gene in JS are nonsense or frameshift mutations, which result in truncated N-terminal AHI1 or loss of WD40 and SH3 domains $(10,11)$. These lossof-function mutations in AHI1 are consistent with the autosomal recessive nature of JS. However, the role of AHI1 in the pathogenesis of JS remains to be elucidated.

Using immunoprecipitation and mass spectrometry, we found that mouse Ahi1 binds tightly to huntingtin-associated protein 1 (Hap1) and forms a stable protein complex in the brain. Hap1 is expressed at variable levels in multiple brain regions $(16,17)$. Mice lacking Hap1 often die at P3, suggesting that Hap1 is critical for neonatal development $(18,19)$. Hap1 is involved in intracellular trafficking and endocytosis of membrane receptors (20-22). Identification of the interaction of Ahi1 with Hap1 prompted us to focus on the role of these proteins in early brain development. Here we demonstrate that Hap1 and Ahi1 stabilize each other and are important for maintaining the level of tyrosine kinase receptor $\mathrm{B}$ (TrkB), which is critical for neuronal differentiation and brain development. The findings in the present study provide evidence that the interaction between Ahi1 and Hap1 is important for the early development of the brain and offer insight into the pathogenesis of JS.

\section{Results}

In vivo association of Abi1 and Hap1. Since the absence of Hap1 leads to the postnatal death of mice $(18,19)$, we wanted to investigate the role of Hap 1 in early brain development by identifying Hap1- 
A

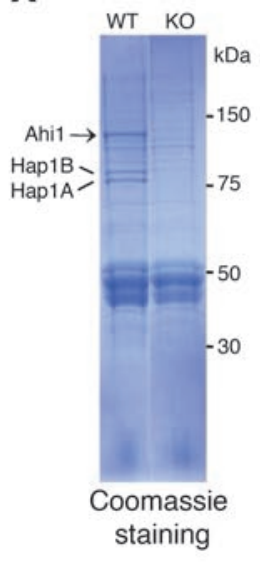

B

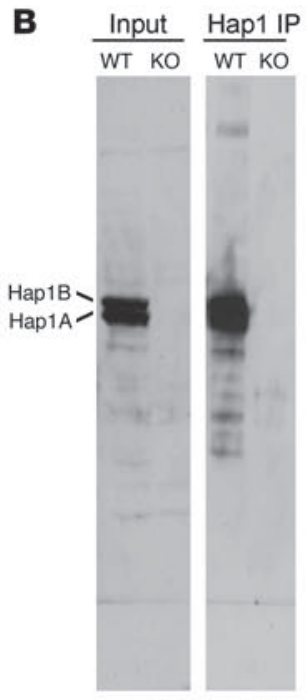

Hap1 blotting

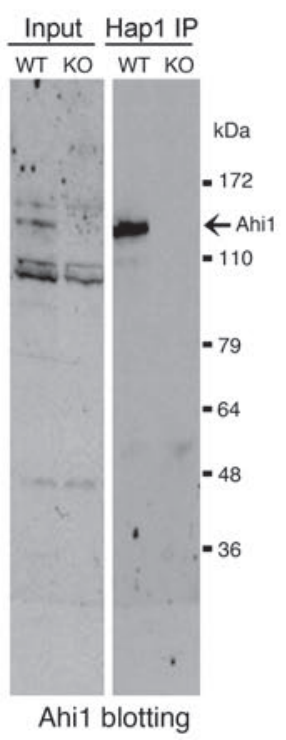

C
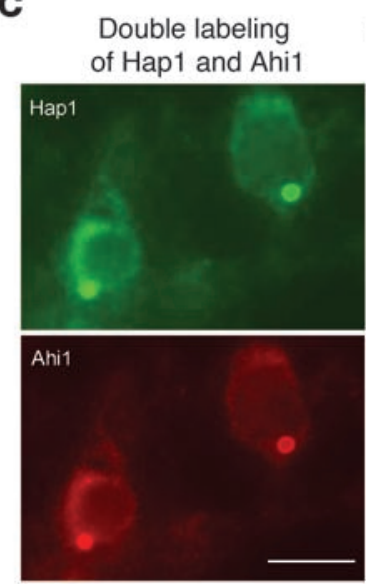

Single labeling of Hap1
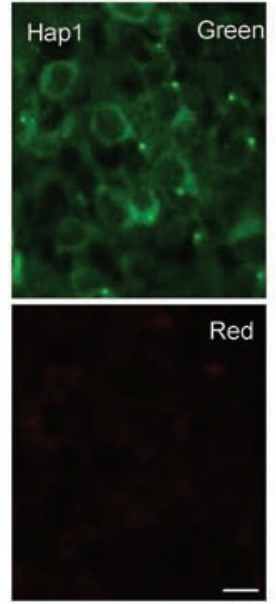

D

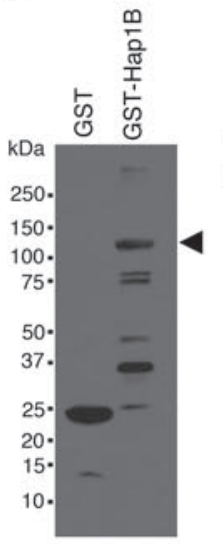

E

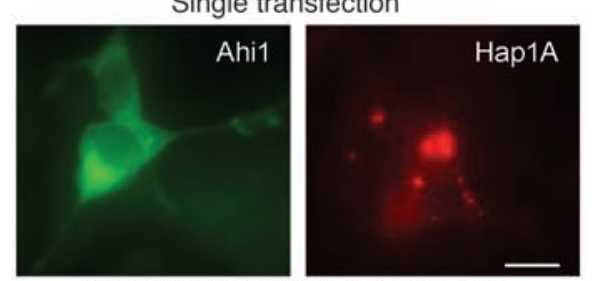

Coexpression of Ahi1 with Hap1A
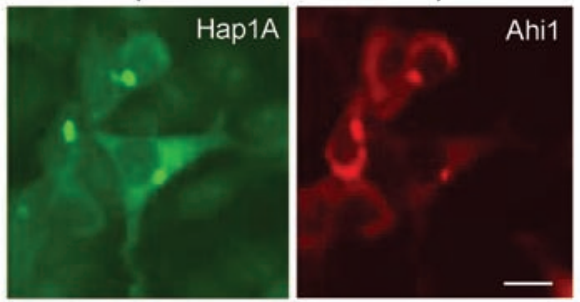

Figure 1

Identification of Ahi1 as a Hap1-interacting protein in the mouse brain. (A) Coomassie staining of Hap1 immunoprecipitates from the brain tissues of WT and Hap1-KO mouse pups. Arrow indicates the band that is present with Hap1A and Hap1B in WT mouse brain tissue. Mass spectrometry peptide analysis identified this band as Ahi1. (B) Western blots of Hap1 immunoprecipitation confirm the coprecipitation of Ahi1 (arrow) with both Hap1A and Hap1B from WT mouse brain tissues. (C) Double immunofluorescence staining of mouse brainstem showing the colocalization of Hap1 and Ahi1 in the cytoplasmic puncta (left panels). Single labeling of the brain section with anti-Hap1 did not show bleeding of the fluorescent signal (right panels). (D) GST and GST-Hap1B were generated, and the intact form of GST-Hap1B is indicated by an arrowhead (left panel). Lysates of HEK293 cells transfected with Ahi1 were pulled down by GST-Hap1 fusion protein. Note that full-length (arrow), but not truncated, Ahi1 bound to Hap1. (E) Cotransfection of Hap1A and Ahi1 in HEK293 cells resulted in the colocalization of both proteins in cytoplasmic puncta. Scale bars: $5 \mu \mathrm{m}$.

interacting proteins in postnatal mouse brain. To this end, we performed immunoprecipitation of cytosolic Hap1 from the brains of WT mice at P1-P2. To identify the interacting proteins that are specifically associated with Hap1, we used Hap1-null mouse brains at P1-P2 as a control and compared the immunoprecipitates from WT and Hap1-null mouse brain tissues. Hap1 consists of 2 isoforms (Hap1A and Hap1B) that differ in their C-terminal amino acid sequences (16). Coomassie staining clearly showed that 2 major bands, which represented Hap1A and Hap1B, were present in the immunoprecipitates from WT but not from Hap1null mouse brains (Figure 1A). Importantly, a prominent band at a molecular weight of $130 \mathrm{kDa}$ was also specifically present in immunoprecipitates from WT mouse brain (arrow in Figure 1A). The abundance of this band in Hap1 immunoprecipitates suggests that the protein in this band is coprecipitated with Hap 1 in near-stoichiometric amounts. The gel slice containing this band was excised and subjected to mass spectrometry peptide sequence analysis. Accordingly, the protein in this band was identified as a mouse homolog (Ahi1) of human AHI1, a protein encoded by a gene for JS $(10,11)$.

Identification of Ahi1 in Hap1 immunoprecipitates led us to generate a rabbit antibody against Ahi1 using glutathione-S-transferase (GST) fusion proteins containing aa 83-174 of mouse Ahi1. This antibody did not show cross-reactivity with Hap1 on West- 
A
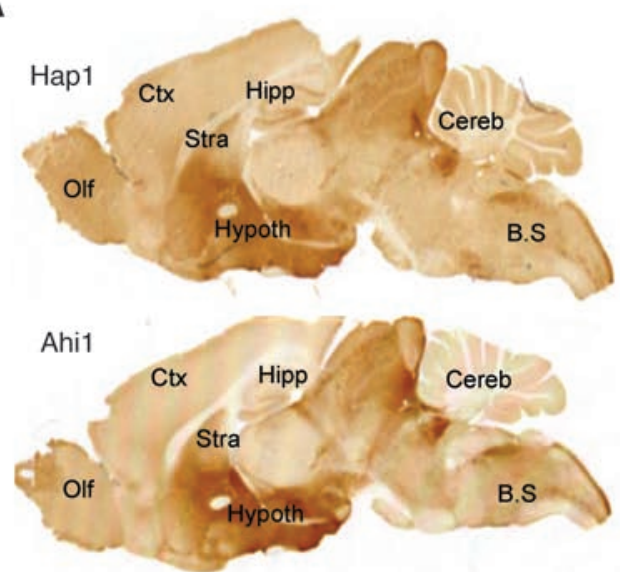

ap1B、

Hap1 $A^{\prime}$

Tubulin

D

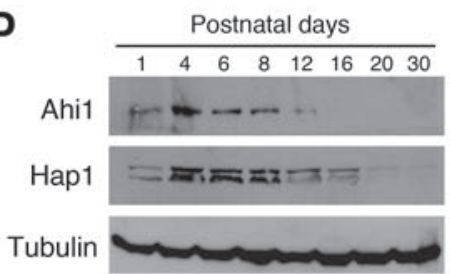

E
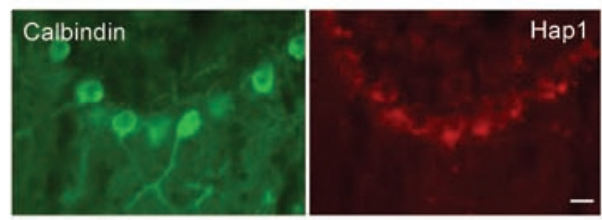

Cerebellum
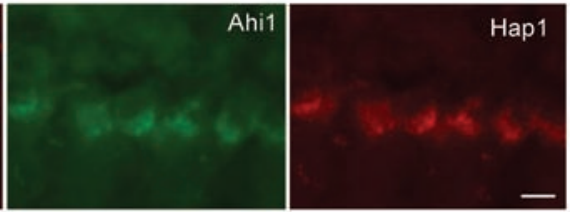

$\mathbf{F}$
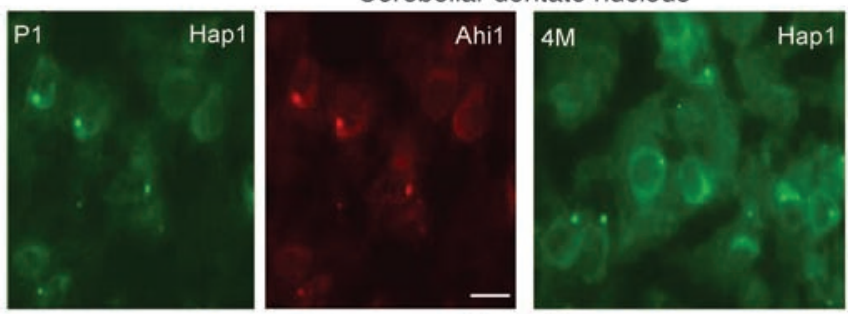

Brainstem
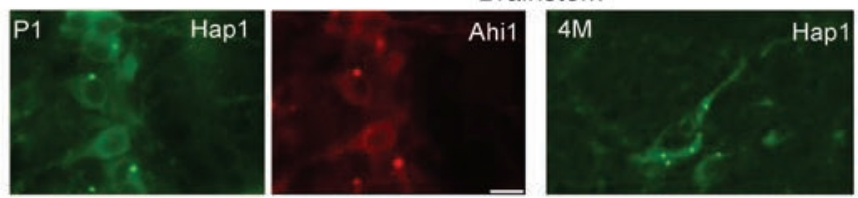
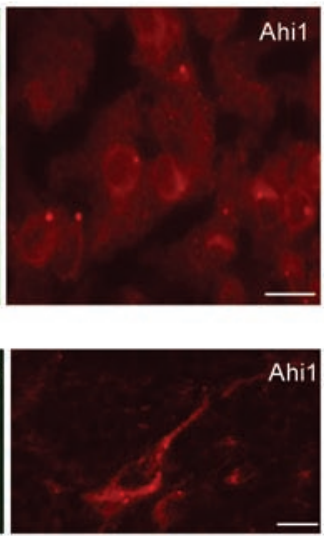

\section{Figure 2}

Hap1 and Ahi1 are distributed in the same brain regions. (A) Immunohistochemical staining of mouse brains with antibodies against Hap1 (top) and Ahi1 (bottom). Olf, olfactory bulb; Ctx, cerebral cortex; Stra, striatum; Hypoth, hypothalamus; Hipp, hippocampus; Cereb, cerebellum; B.S, brainstem. (B) Western blots showing the similar distribution of Hap1 and Ahi1 in various brain regions. Amy, amygdala. (C) Western blot analysis of the expression of Hap1 and Ahi1 in embryonic mouse brains. Two mouse brains ( 1 and 2 ) at E8, E12, and E18 were examined. (D) Western blots of the cerebellar tissue from mice at different postnatal days (days 1-30). The blots were probed with antibodies against Ahi1, Hap1, and tubulin. (E) Immunostaining of the adult mouse cerebellum with antibodies against calbindin and Hap1 together or antibodies against Ahi1 and Hap1 together. (F) Immunofluorescence staining showing that Hap1 and Ahi1 are colocalized in the same neurons in the deep dental nuclei in the cerebellum (top row) and brainstem (bottom row) from mice at P1 and at 4 months (4M) of age. Scale bars: $5 \mu \mathrm{m}$. ern blot analysis and confirmed that Ahi1 was coprecipitated with Hap1 from mouse brain lysates (Figure 1B).

Since Hap1 is present as cytoplasmic puncta in the brain (17), we wanted to determine whether Ahi1 and Hap1 colocalize in vivo. Hap1 cytoplasmic puncta in brainstem neurons were intensely labeled by a guinea pig antibody against Hap1. Importantly, the same Hap1 puncta were also labeled by the rabbit antibody against Ahi1 (Figure 1C).

To test whether Hap1 and Ahi1 interact directly, we performed a GST pull-down experiment. Expression of mouse full-length Ahi1 cDNA in HEK293 cells generated full-length Ahi1 and number of degraded products, which were recognized by the antibody to $\mathrm{N}$-terminal Ahi1 (aa 83-174). We found that GST fusion proteins containing full-length Hap1B bound to full-length, but not truncated, Ahi1 (Figure 1D). Using in vitro synthesized Hap1A or Hap1B, we also found that GST-Ahi1 directly bound Hap1A and Hap1B (Supplemental Figure 1; supplemental material available online with this article; doi:10.1172/JCI35339DS1). Single immunostaining of the mouse brain sections with the antibody against Hap1 or Ahi1 alone did not yield bleeding signal between green and red fluorescence filters (Figure 1C and Supplemental Figure 2A). Cotransfection of Ahi1 with Hap1A, which forms cytoplasmic puncta, clearly revealed that Ahi1 colocalized with Hap1A (Figure 1E and Supplemental Figure 2B). Interestingly, although Hap1B is diffuse in the cytoplasm when singly transfected (22, $23)$, its cotransfection with Ahi1 resulted in small puncta in which Hap1B and Ahi1 were colocalized (Supplemental Figure 2B). Immunostaining of transfected proteins also demonstrated the specificity of the antibodies used for Hap1 and Ahi1.

Coexpression of Hap1 and Abi1 in the same neurons in various brain regions. We have previously shown that Hap1 expression levels vary 
A

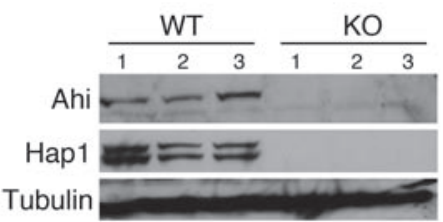

B WT KO
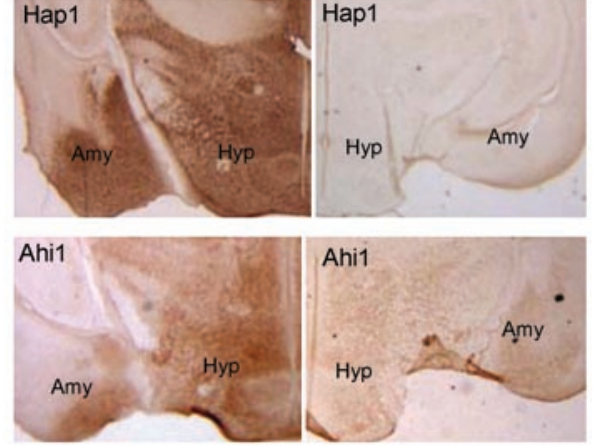

C

WT
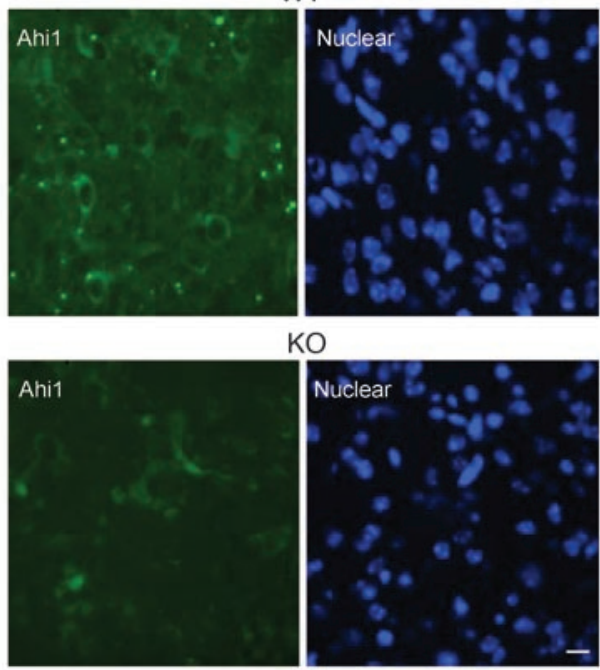

$\mathrm{KO}$

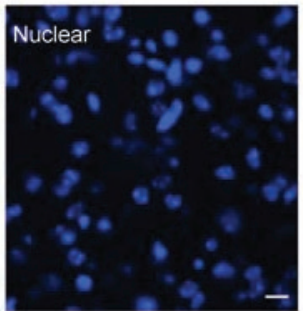

D

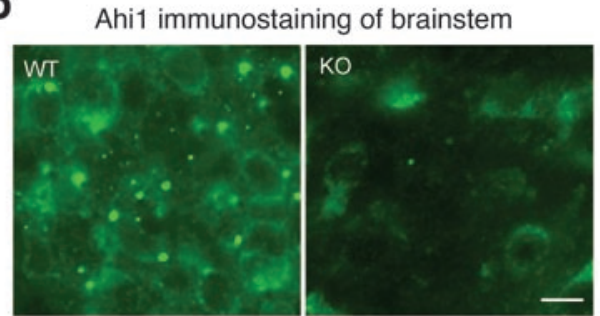

E
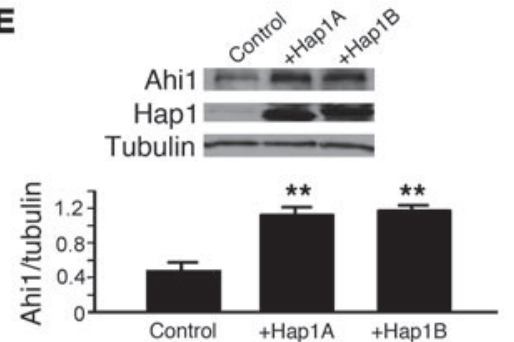

$\mathbf{F}$

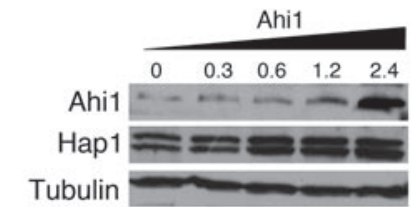

G
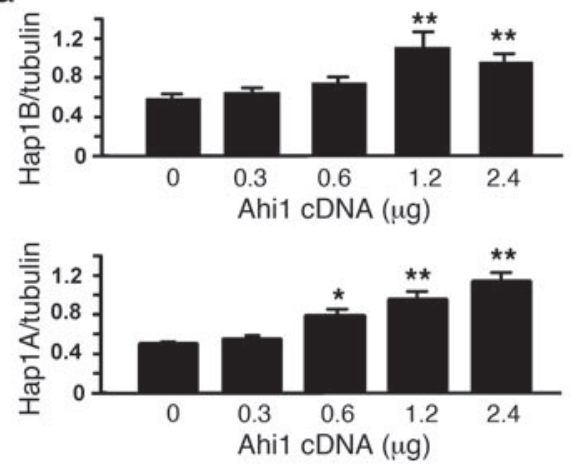

\section{Figure 3}

Hap1 and Ahi1 stabilize each other. (A) Western blots showing that a lack of Hap1 reduces the level of Ahi1 in Hap1$\mathrm{KO}$ mouse brain. (B) Immunostaining of the hypothalamus (Hyp) and amygdala (Amy) from WT and Hap1-KO mice also shows a decreased level of Ahi1 in the absence of Hap1. Original magnification, $\times 50$. (C and $\mathbf{D}$ ) Immunofluorescence images showing the lack of cytoplasmic Ahi1 puncta in neurons of the hypothalamus (C) and brainstem (D) in Hap1-KO mice. Scale bars: $5 \mu \mathrm{m}$. (E) Transfection of Hap1A or Hap1B into N2A cells increases the level of endogenous Ahi1. The relative levels (mean \pm SEM; $n=4$ ) of Ahi1 (the ratio of Ahi1 to tubulin on the same blot) are presented below the blots. ${ }^{* \star} P<0.01$ compared with control. (F) Overexpression of different doses of Ahi1 in PC12 cells. Note that increasing Ahi1 expression also increases the level of endogenous Hap1. (G) The ratio (mean $\pm \mathrm{SEM} ; n=3$ ) of Hap $1 \mathrm{~A}$ or Hap1B to tubulin in cells transfected with different amounts $(0.3-2.4 \mu \mathrm{g})$ of Ahi1 cDNA. ${ }^{*} P<0.05,{ }^{* *} P<0.01 \mathrm{com}-$ pared with nontransfected cells $(0 \mu \mathrm{g})$. in different brain regions $(16,19)$. If Ahi1 forms a stable complex with Hap1, its distribution pattern may be the same as that of Hap1. Indeed, immunohistochemistry demonstrated that Ahi1 and Hap 1 are distributed in the same brain regions, with the highest expression seen in the hypothalamus, amygdala, and brainstem (Figure 2A). Western blotting also revealed this similar distribution in various brain regions (Figure $2 \mathrm{~B}$ ), supporting the idea that Hap1 and Ahi1 are strongly associated.

Since mutations of Ahi1 cause abnormal development of the cerebellum and brainstem in JS, we investigated the expression of Ahi1 and Hap1 in these brain regions during early development. Previous studies using in situ hybridization have demonstrated that Hap1 and Ahi1 transcripts are developmentally regulated, appearing at E8 and becoming prominent at E12 (10, 11, 24). We examined the expression of Hap 1 and Ahi1 at the protein level and found that both Ahi1 and Hap 1 proteins, though undetectable at E8, appear at E12 and have increased levels by E18 (Figure 2C).

The final structure of the mature cerebellum in mice is not achieved until approximately P15 (25). Western blotting detected
Hap1 and Ahi1 in the cerebellum on P1-P12, but not after P16 (Figure 2D). Immunohistochemistry of mouse brains revealed that the expression of both Hap1 and Ahi1 is restricted to Purkinje cells (Figure 2E and Supplemental Figure 3A). They were also expressed in the deep cerebellar dentate nuclei (Figure $2 \mathrm{~F}$ ), interposed cerebellar nuclei, medial cerebellar nuclei, and vestibular nuclei (Supplemental Figure 3B). Granule neurons and glial cells do not show obvious staining of Hap1 and Ahi1. As the mature cerebellum of adult mice is dominated by granule neurons and other cells that do not display Hap1 and Ahi1 immunostaining, the expression of Hap 1 and Ahi1 in the adult cerebellum is restricted to Purkinje cells and cerebellar nuclei, which could be identified by immunohistochemistry.

The brainstem is another area affected in JS. We found that Hap1 and Ahi1 are also expressed in the same neurons in the brainstem. Their expression in the brainstem was present in postnatal (P1) and adult mice at 4 months of age (Figure 2F).

Abi1 and Hap1 stabilize each other. The strength of the association of Ahi1 with Hap1 implies that this association may be important 
A

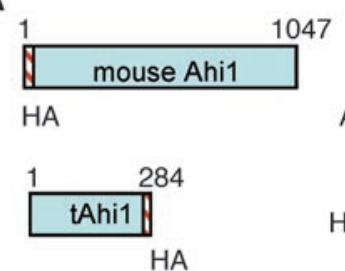

HA

tAhi1
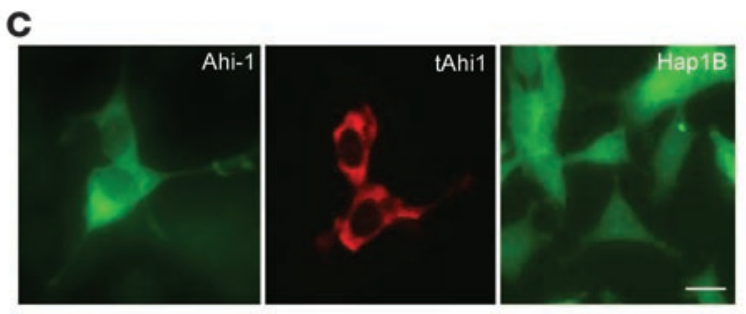

Coexpression of Ahi1 and Hap1B

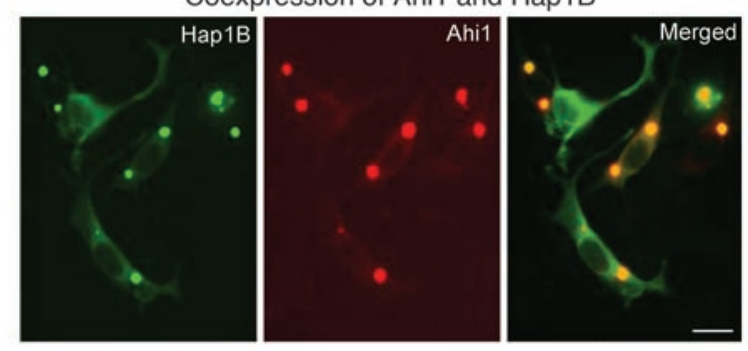

Coexpression of tAhi1 and Hap1B
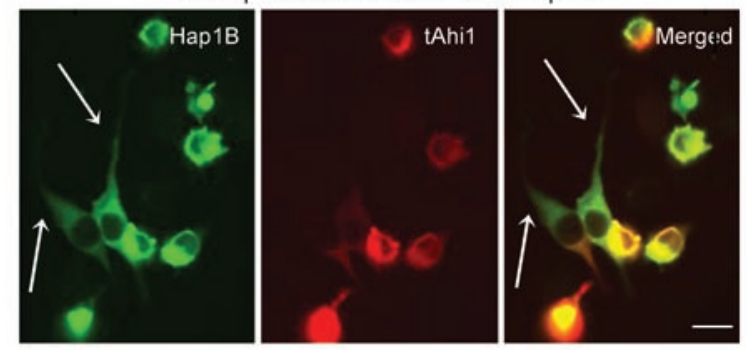

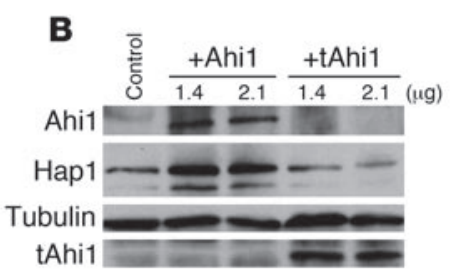

D
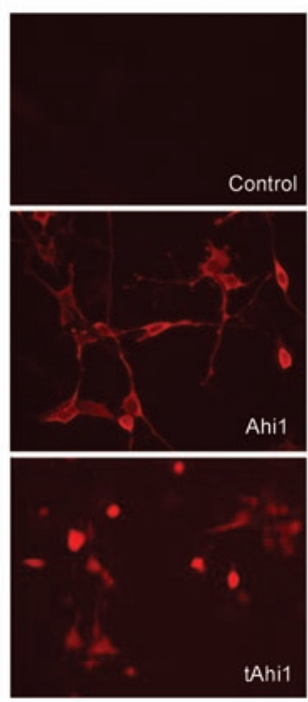

E

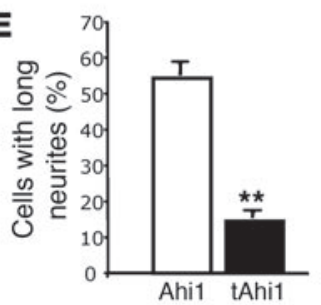

\section{Figure 4}

Truncated Ahi1 fails to increase Hap1 expression. (A) Full-length or truncated mouse Ahi1 was cotransfected with Hap1B and then immunoprecipitated by anti-Hap1. Note that more full-length Ahi1 than truncated Ahi1 was coprecipitated with Hap1. (B) Overexpression of fulllength or truncated Ahi1 in PC12 cells. Only full-length, but not truncated, Ahi1 was able to increase the level of endogenous Hap1. (C) Expression of Ahi1 or Hap1B alone in PC12 cells resulted in a diffuse distribution of transfected proteins (top row). Coexpression of Hap1B and full-length, but not truncated, Ahi1 led to the formation of small cytoplasmic puncta. Note that truncated Ahi1 also inhibited NGF-induced neurite extension of PC12 cells (bottom row) compared with those coexpressing both Hap1B and full-length Ahi1 (middle row). Scale bars: $5 \mu \mathrm{m}$. (D) Transfection of full-length or truncated Ahi1 alone into $\mathrm{PC} 12$ cells that were treated with NGF (100 ng/ml for 24 hours). Micrographs $(\times 200)$ show that truncated Ahi1 inhibits neurite outgrowth of PC12 cells. (E) The percentage of $\mathrm{PC} 12$ cells with elongated neurites longer than 2 cell bodies after transfection with full-length or truncated Ahi1. ${ }^{\star *} P<0.01$. for their stability. Examination of several Hap1-null mice revealed a significant reduction in Ahi1 protein in their brains compared with those of WT mice (Figure 3A). While both Hap1 and Ahi1 were abundant in the hypothalamus and amygdala, elimination of Hap1 dramatically reduced the expression of Ahi1 in these regions (Figure 3B). Moreover, Hap1-KO mice did not show hypothalamic Ahi1 puncta, which are normally present in WT mice (Figure 3C). Also, cytoplasmic Ahi1 puncta disappear in the brainstem of Hap1KO mice (Figure 3D).

The above in vivo data strongly suggest that Hap 1 and Ahi1 form a protein complex to stabilize each other. If this is true, then overexpression of Hap1 or Ahi1 should increase the level of its partner. Thus, we transfected Hap1 or Ahi1 into N2 cells, a mouse neuroblastoma cell line that allows us to use anti-Ahi1 to detect endogenous mouse Ahi1. Overexpression of either Hap1A or Hap1B increased the level of endogenous Ahi1 (Figure 3E). Using anti-Hap1 that recognizes endogenous Hap1 in PC12 cells of rat origin, we found that transfected Ahi1 increased the level of endogenous Hap1A and Hap1B in a dose-dependent manner (Figure 3, F and G).
Truncated Abi1 is unable to stabilize Hap1. Given that most mutations in human AHI1 result in truncated proteins that often contain $\mathrm{N}$-terminal sequences shorter than the first 435 aa, we asked whether truncated Ahi1 loses its ability to stabilize Hap1. Human AHI1 contains more N-terminal amino acids than mouse Ahi1 (13). We generated a truncated mouse Ahi1 (1-284 aa) corresponding to the nonsense mutation at amino acid 435 of human AHI1 and examined its association with Hap1 (Figure 4A). Consistent with GST pull-down results (Figure 1D), immunoprecipitation of transfected Hap1 coprecipitated more full-length Ahi1 than truncated Ahi1 in cultured HEK293 cells (Figure 4A). Moreover, unlike full-length Ahi1, truncated Ahi1 did not increase the level of endogenous Hap1 in PC12 cells (Figure 4B). Because of the presence of endogenous Ahi1 that has already formed the stable complex with endogenous Hap1, overexpression of exogenous truncated Ahi 1 affected the stability of Hap1 only slightly. Since coexpression of Hap1B with full-length Ahi1 leads to the formation of small cytoplasmic puncta in cells (Supplemental Figure 2B), we examined whether truncated Ahi1 loses this ability in 
A

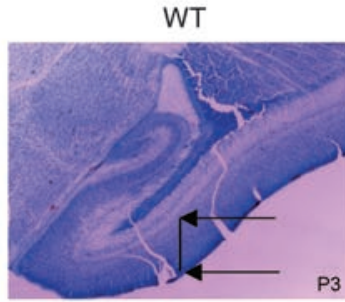

KO

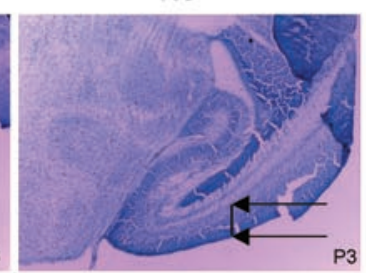

KO
B

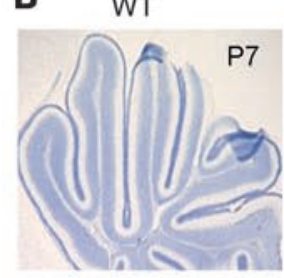

P7

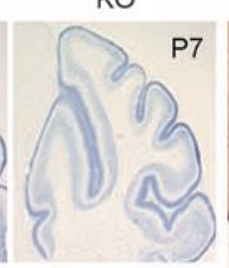

P7

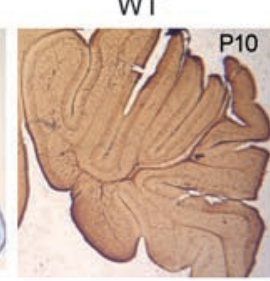

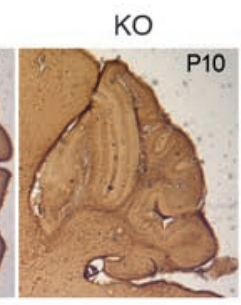

C

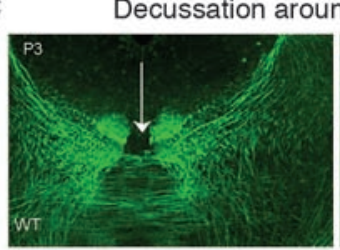

Decussation of superior cerebellar peduncle
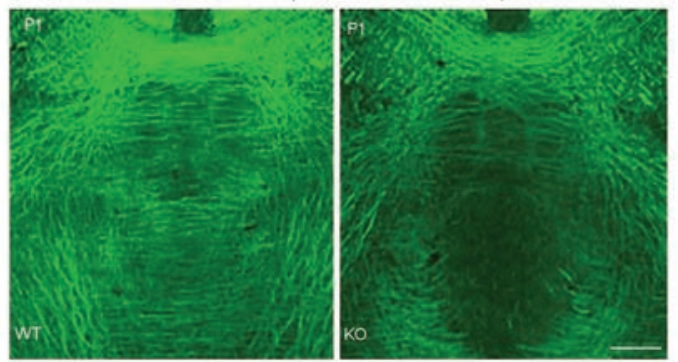

\section{Figure 5}

Lack of Hap1 causes abnormal brain development. (A) Nissl staining of the cerebellar sections from WT and Hap1-KO mice. Arrows indicate the width of the outer cerebellar cortex in the folium of vermal lobule V in WT and KO mice at P3. Original magnification, $\times 100$. (B) Comparison of cerebellar development of WT and Hap1-KO mice at P7 and P10. Original magnification, $\times 50$. (C) Representative images reveal defective decussation visible around the $3 \mathrm{~N}$ nucleus and the superior cerebellar peduncle in Hap1-KO cerebellum. Such defective decussation was never observed in age-matched WT littermates. The brain sections were stained with the antibody against neurofilament. Scale bars: $50 \mu \mathrm{m}$.

neuronal PC12 cells. Indeed, unlike full-length Ahi1, truncated Ahi1 failed to form small cytoplasmic puncta with Hap1 (Figure 4C). In addition, expression of truncated Ahi1 with Hap1B inhibited neurite outgrowth of PC12 cells compared with cells coexpressing full-length Ahil. To confirm this inhibitory effect, we transfected full-length or truncated Ahi1 alone into PC12 cells (Figure 4D). Counting of the transfected cells revealed that $55.3 \% \pm 3.5 \%$ of full-length Ahi1 and $15 \% \pm 2.1 \%$ (mean \pm SEM; $P<0.01$ ) of truncated Ahi1 transfected cells showed long neurites (Figure 4E), indicating that truncated Ahi1 significantly inhibits nerve growth factor-induced (NGF-induced) neurite outgrowth. We tested the effects of truncated human AHI1 (aa 1-435) and found that this human mutant AHI1 also inhibited NGF-induced neurite outgrowth in PC12 cells (data not shown). All of these results suggest that truncated Ahi1 is unable to stabilize Hap1 and, when overexpressed, has a toxic effect.

Abnormal brain development in the absence of Hap1. Identification of Ahi1 as a Hap1-interacting partner led us to investigate whether Hap1-null mice display any developmental brain abnormalities similar to those seen in JS. Since the postnatal lethality of Hap1null mice does not allow us to examine the structure of the mature brain and cerebellum, we focused on possible malformation of the cerebellum and brainstem during early postnatal development. There was a reduction in the size of cerebellar folia in Hap1-null mice compared with littermate controls (Figure 5A). Very few Hap1-KO mice can survive past P3, and all die before P15 (19). In those few surviving mice at P7 and P10, the reduction in cerebellar foliation was more pronounced (Figure 5B). In addition, the density of the calbindin-positive Purkinje cells and the number of NeuN-positive granule cells are noticeably decreased in the Hap1KO cerebellum (Supplemental Figure 4A).

Since Hap 1 is not expressed in granule cells but is important for intracellular transport $(22,23,26)$, lack of Hap1 may affect neuronal interactions that are required for the massive growth of the cerebellum. Indeed, axonal defects account for the abnormal decussation that is the most prominent pathological feature of JS. Using neurofilament staining, we observed decreased decussation around the $3 \mathrm{~N}$ (oculomotor) nucleus and the superior cerebellar peduncles (Figure 5C) in Hap1-null mice at P1-P2. The abnormal decussation appears as disrupted neurofilaments.

If a lack of Hap1 affects early brain development, we may see impairment of neurogenesis in postnatal brains. Neurogenesis can be detected by BrdU labeling of brain tissues (27). We therefore examined the incorporation of BrdU in the deep cerebellar nuclei and brainstem, 2 regions that express Hap 1 and Ahi1 and are affected in JS. We observed a significant reduction in the number of BrdU-positive cells in the absence of Hap1 (Supplemental Figure 4B). These results also support the idea that Hap1 is important for neuronal differentiation and proliferation.

Hap1 is required for TrkB signaling. Hap1 prevents some internalized membrane receptors from being transported to the lysosome for degradation $(20,21)$ and stabilizes the level of internalized TrkA (20). TrkB is similar in structure to TrkA, but it is more abundant than TrkA in the brain $(28,29)$ and is activated by brainderived neurotrophic factor (BDNF), a growth factor that is critical for neurogenesis and cerebellar development $(30,31)$. Thus, we examined whether the lack of Hap1 also affects TrkB levels in the postnatal mouse brain using Western blotting, as Western blotting had detected both Ahi1 and Hap1 in cerebellar tissues of WT mice between P1 and P8 (Figure 2D). The level of TrkB is significantly reduced in cerebellar tissues from Hap1-KO mice aged P1-P2 compared with age-matched WT mice (Figure 6A). In the brainstem, we also found that a lack of Hap1 reduces Ahi1 and TrkB (Figure $6 \mathrm{~A}$ ). Densitometry of the relative levels of TrkB (the ratio of TrkB to tubulin) confirmed the decreased levels of TrkB in Hap1-KO mouse brains (Figure 6B). Since JS is likely caused by Ahi1 loss of function, we wanted to know whether suppressing Ahi1 reduces TrkB in cerebellar neurons. To inhibit Ahi1 expression, we cultured 


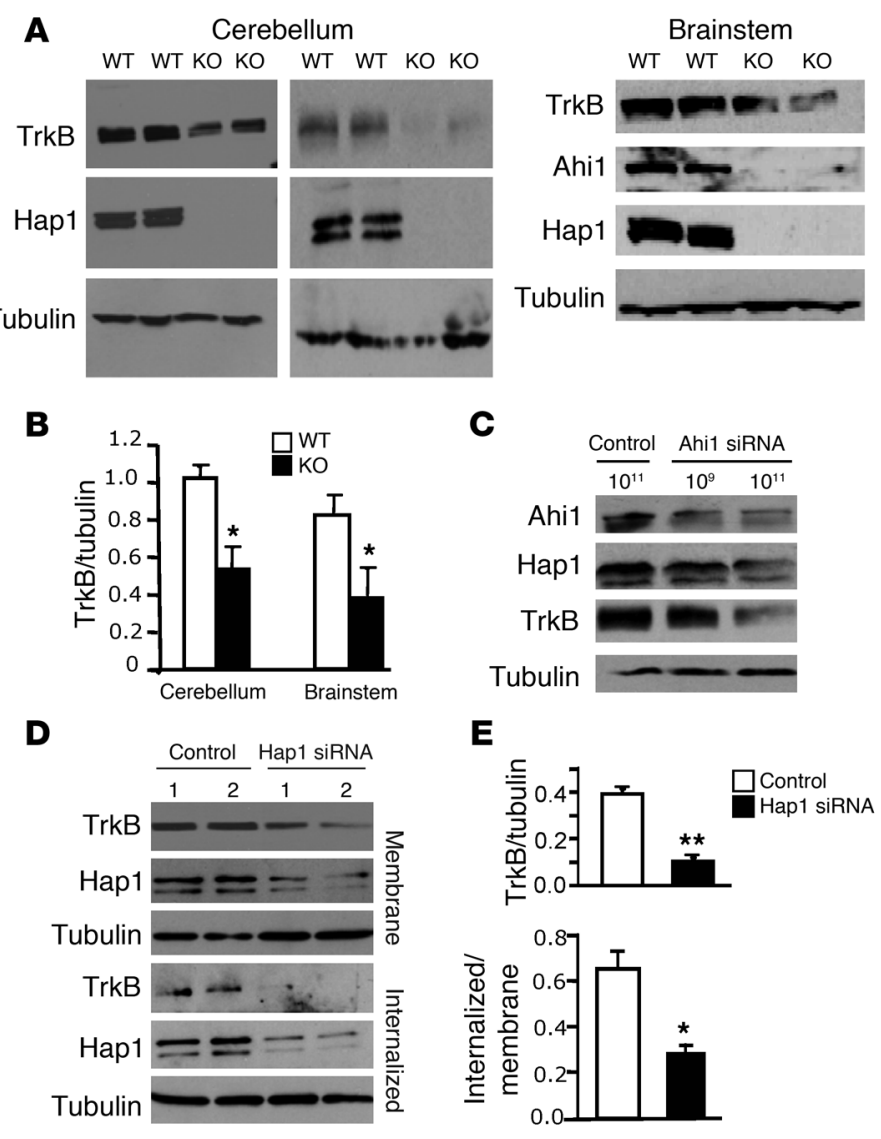

\section{Figure 6}

Hap1 deficiency decreases the level of TrkB. (A) Western blot analysis of cerebellar tissues from WT and Hap1-KO mice showing the decreased level of TrkB. Western blotting also shows decreased Ahi1 and TrkB in the absence of Hap1 in brainstem tissue. (B) Densitometry of the ratio of TrkB to tubulin in WT and $\mathrm{KO}$ cerebellum or brainstem. ${ }^{*} P<0.05$. (C) Inhibition of Ahi1 in cultured cerebellar neurons by adenoviral siRNA ( $10^{9}$ and $10^{11}$ $\mathrm{PFU} / \mathrm{ml}$ at final concentration) reduced the levels of Hap1 and TrkB. Control was adenoviral GFP infection. (D) Western blotting of biotinylated membrane and internalized fractions in cultured brainstem cells showing the decreased level of TrkB when Hap1 expression is suppressed by Hap1 siRNA. Control is adenoviral GFP-infected cells. The blots were probed with an antibody against tubulin to normalize the protein amount loaded in the gel. (E) The ratios (mean \pm SEM; $n=4$ ) of internalized TrkB to tubulin or membrane TrkB in neurons infected with control or Hap1 siRNA virus. ${ }^{*} P<0.05,{ }^{* *} P<0.01$ compared with control. cerebellar neurons from postnatal mouse brains and infected them with adenoviral Ahi1 siRNA. Western blots show that reducing Ahi1 levels in these neurons decreased the expression of Hap1 and TrkB (Figure 6C). Taken together, our results demonstrate that loss of either Hap1 or Ahi1 can reduce the level of TrkB.

Next, we investigated the effect of Hap1 deficiency on TrkB levels in cultured brainstem cells. Using cultured WT rat brainstem cells, we suppressed Hap1 expression via an adenoviral Hap1 siRNA vector that also expressed GFP (32). We then performed biotinylation of membrane receptors and isolated membrane and internalized fractions of brainstem cells to examine the relative levels of TrkB (33). Although the level of membrane TrkB was indeed decreased, internalized TrkB was also reduced (Figure 6D), and this was confirmed by quantifying the ratio of internalized TrkB to tubulin or to membrane TrkB and comparing it with the ratio in control cells (Figure $6 \mathrm{E})$. Consistently, suppressing Hap1 via siRNA inhibited neurite extension of cultured brainstem cells (Supplemental Figure 5).

To substantiate the finding of decreased BDNF in Hap1-deficient neurons, we performed fluorescence microcopy of cultured brainstem cells. We observed that neurons infected with Hap1 siRNA virus showed decreased signals of internalized BDNF compared with those infected with control GFP virus (Figure 7A). Using cultured brainstem cells from Hap1-KO pups, we also observed a significant reduction in internalized BDNF in Hap1$\mathrm{KO}$ neurons (Figure 7B). Quantifying the cytoplasmic intensity of BDNF revealed a decrease in internalized BDNF signal in Hap1KO (413 \pm 156.2 versus WT, $868 \pm 139$; mean \pm SEM; $n=28-33$; $P<0.001)$ and Hap 1 siRNA-treated neurons $(475 \pm 38.3$ versus control, $819 \pm 82.6$; mean \pm SEM; $n=32-35 ; P<0.001)$.
If TrkB levels are reduced by decreasing Hap 1 in cells, the signaling pathways mediated by TrkB should be impaired. We therefore examined the phosphorylation of Erk and Akt, both of which are key signaling molecules for neuronal differentiation and survival. Suppressing Hap 1 expression by siRNA also reduced the level of phosphorylated Erk and Akt (Figure 7C). Quantification of the ratio of phosphorylated Erk or Akt to the total amount of Erk and Akt confirmed the decreased phosphorylation of these proteins (Figure 7D). Taken together, our results indicate that Hap1 is important for maintaining the level and signaling of TrkB in neuronal cells.

\section{Discussion}

Stable interaction of Abi1 with Hap1 in vivo. Several lines of evidence indicate that Ahi1 and Hap1 form a stable protein complex in vivo. First, immunoprecipitation of Hap1 from mouse brain tissue revealed that similar amounts of Ahi1 and Hap1 were coprecipitated, suggesting that they are associated with roughly equal stoichiometry. Second, both Hap1 and Ahi1 colocalize in cytoplasmic puncta in the brain and in transfected cells. More importantly, when Hap1 is absent, as in Hap1-KO mouse brain, the level of Ahi1 significantly decreases, and it no longer appears as punctate structures. Conversely, suppressing Ahi1 via siRNA reduces Hap1 in cultured neurons. Finally, overexpression of either Hap1 or Ahi1 can increase the level of its partner.

There is growing evidence that Hap1 is involved in intracellular trafficking. For example, Hap1 interacts with the microtubuledependent transporters dynactin p150 (34) and kinesin light chain (35) and is involved in the internalization of several membrane 
A
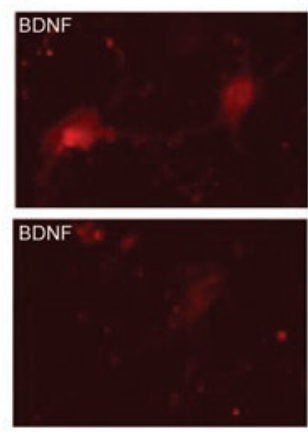

Cultured rat brainstem cells
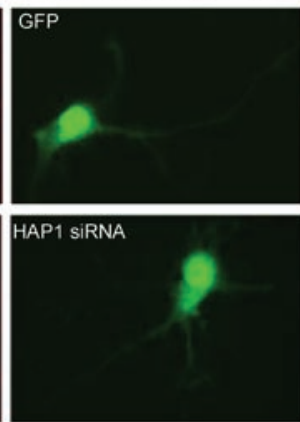

B

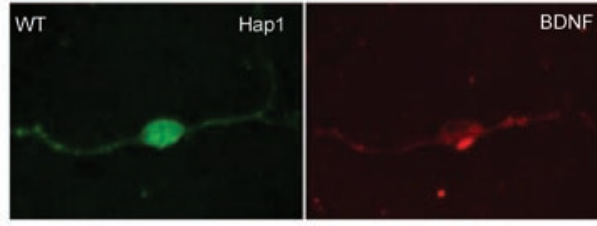

Cultured mouse brainstem cells
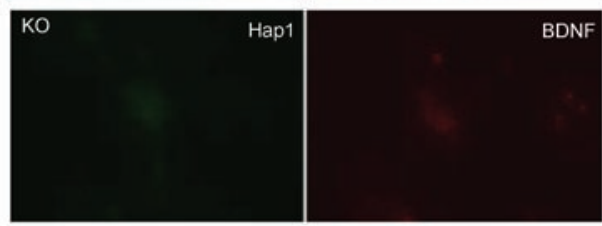

BDNF
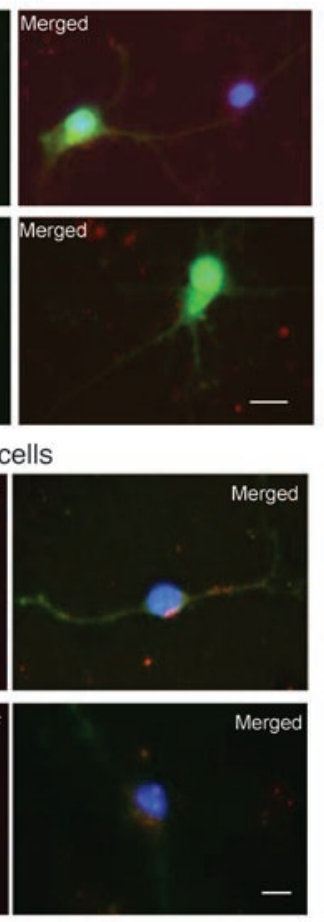

C

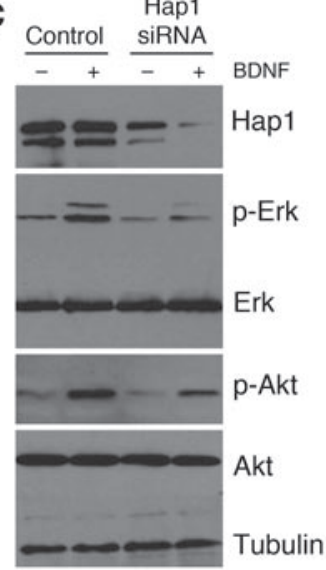

D

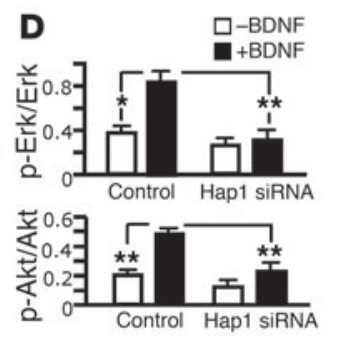

\section{Figure 7}

Hap1 deficiency decreases TrkB internalization and signaling. (A) Double immunostaining of cultured brainstem cells (at 14 DIV) that had been infected with adenoviral GFP (top row) or adenoviral Hap1 siRNA with GFP (bottom row). Note that cells expressing Hap1 siRNA (green) show a decrease in the amount of internalized biotin-labeled BDNF compared with a noninfected cell or adenoviral GFP-infected cell. (B) Double immunostaining of cultured brainstem cells from WT (top row) and Hap1-KO (bottom row) pups also shows the decreased Hap1 and internalization of biotin-BDNF. The cells were stained with rabbit anti-Hap1 and mouse anti-biotin. Statistical results are described in the text. Scale bars in A and B: $5 \mu \mathrm{m}$. (C) Decreased phosphorylation of Erk and Akt in cultured brainstem cells infected by adenoviral Hap1 siRNA. The blots were probed with antibodies against Akt, Erk, and their phosphorylated forms. (D) The ratio (mean \pm SEM; $n=3-4$ ) of phosphorylated Erk or Akt to total Erk or Akt was quantified by densitometry. ${ }^{*} P<0.05,{ }^{* *} P<0.01$.

sion in cultured neuronal cells. Since JS results from the loss of Ahi1, it is important to use a gene-targeting approach to investigate whether this toxicity can be caused by truncated Ahi1 at the endogenous level.

The expression of Abil and Hap1 and early brain development. The extensive postnatal proliferation of the external granular layer and subsequent migration of differentiating granule neural cells result in massive growth of the cerebellum (37). In addition, foliation of the cerebellum is closely associated with ingrowth of cerebellar afferents (38). The absence of Hap1 leads to reduced foliation of the cerebellum in Hap1-null mice, suggesting that Hap1 is required for cerebellar development. The cerebellum is known to control body movement and balance. Although Hap1-null mice often die at P3, some of these mice can survive until P15. In those rare surviving mice, ataxia and movement imbalances were observed (data not shown), also suggesting that a lack of Hap1 can affect cerebellar function. However, the expression of Hap 1 and Ahi1 is not widespread in the cerebellum, but rather restricted to the cerebellar nuclei and Purkinje cells. The

receptors, including $\mathrm{GABA}_{\mathrm{A}}(21)$ and $\operatorname{TrkA}(22)$ receptors. Given that Hap 1 interacts with a variety of proteins in various subcellular regions, further studies will be required to determine whether Ahi1 can regulate the association of Hap1 with other proteins. Also, it would be interesting to examine whether coprecipitated proteins with Ahi1 are diminished or vanish in Hap1-KO mouse brain.

The colocalization of Hap1 and Ahi1 in cytoplasmic puncta also suggests that these 2 proteins form a stable complex in cells. Although the function of this structure remains unknown, it is identical to that of the previously identified stigmoid bodies, which are cytoplasmic structures seen in neurons in the hypothalamus, thalamus, amygdala, septum, hippocampus, and brainstem (36). Our findings reveal the possibility that these normal cytoplasmic puncta in neurons may be important for the assembly of the Hap1-Ahi1 complex, since eliminating Hap1 prevents the appearance of Ahi1-immunoreactive puncta. It is possible that the Hap1-Ahi1 complex stabilizes both proteins or prevents their degradation to maintain their normal levels and function. Accordingly, loss of one of the proteins reduces the stability of its partner and subsequently affects the function of this protein complex. In addition, truncated Ahi1 can likely induce a toxic gain of function, as overexpression of truncated Ahi1 could suppress neurite exten- structural relationships of 4 deep cerebellar nuclei (the dentate, emboliform, globose, and fastigial) are generally maintained in the neuronal connections between the nuclei and associated cerebellar cortex (39). For example, in late embryogenesis, climbing fibers from the inferior olivary nucleus, which are innervated by deep cerebellar neurons, start to innervate Purkinje cells that control the proliferation of granule neurons via the release of diffusible factors (37-40). Decreasing the expression of Hap1 and Ahi1 could affect the function of these output neurons and impair the cerebellar network or the input that is required for maturation and formation of the cerebellar structure. It should be noted as well that the ventricular zone produces Purkinje cells, which are also important for cerebellar development. Loss of Hap1 and Ahi1 could affect the function of these neurons and therefore could affect neurogenesis in the cerebellum.

Hap1 and Ahi1 are expressed in various brain regions. In fact, Hap1 is more abundant in the hypothalamus, and Hap1-null mice show degeneration of hypothalamic neurons and a feeding defect $(19,32)$. Although Ahi1 and Hap1 form a stable protein complex in many brain regions, their distribution could be different in some cells, where they may interact with unique partners and mediate different functions. For example, Ahi1 is found in blood cells (13), 
whereas Hap1 is seen in endocrine cells (41). It is possible that the mutations of Ahi1 in JS cause a partial loss of Hap1 function, which leads to a more selective defect in the cerebellum and brainstem than the severe effect caused by complete depletion of Hap1.

The function of Abi1 and the pathogenesis of JS. Mouse Abi1 was initially found to be involved in oncongenic leukemia, as Abelson murine leukemia virus-induced pre-B lymphomas harbor a provirus inserted within the Abi1 locus $(12,13)$. Consistently, tumor cells harboring insertional mutations in Abil show truncated Abi1/ viral fused transcripts (13). Germline mutations in AHI1 could cause abnormalities in early brain development, whereas somatic mutations of AHI1 due to viral insertions lead to oncogenic events in hematopoietic cells. AHI1 contains an SH3 domain and WD40 regions, which are found in many proteins that participate in cell signaling and intracellular trafficking. The abnormal decussation seen in JS reflects defective axonal crossing due to abnormal axonal guidance or neuronal differentiation. The finding that Ahi1 binds to Hap1, which is involved in intracellular trafficking and Trk receptor internalization, fits with the idea that Ahi1 deficiency in neurons can affect neuronal interaction and networks.

Hap1 is known to be transported in axons and is important for neurite outgrowth $(22,23,26)$. There is mounting evidence that Hap 1 is also involved in the internalization of membrane receptors. For example, Hap1 stabilizes the level of internalized receptors, such as EGF (20), GABA $(21)$, and TrkA (22) receptors. Hap1 may interact with microtubule-dependent transporters to participate in the internalization, trafficking, and recycling of various membrane receptors, and its function probably depends on the levels of its partners in a given type of neuron. TrkB has a structure similar to that of TrkA but is more abundant in the cerebellum and brainstem, whereas TrkA is more restricted to sensory and sympathetic neurons $(28,29)$. Activation of TrkB by BDNF leads to the internalization of $\operatorname{TrkB}$ receptors, which brings activated $\operatorname{TrkB}$ receptors to the nucleus to trigger gene transcription and to membrane compartments to mediate signaling cascades (42). BDNF is an important modulator of synaptic plasticity, and TrkB receptor signaling is required for establishment of GABAergic synapses in the cerebellum (43). Mice lacking TrkB die postnatally, due to depressed feeding behavior (44), a defect that is also seen in Hap1-null mice $(18,19)$. Although the brains of mutant mice deficient in a single Trk receptor species do not show signs of major cell loss, loss of TrkB and TrkC receptors can reduce the number of postnatal cerebellar granule cells and causes poor differentiation of cerebellar Purkinje cells (45). All of these previous findings suggest that TrkB is important for the maturation of neurons in the cerebellum.

Our studies revealed that a lack of Hap1 or suppression of its levels could decrease the level of TrkB. Since Hap1 has been found to prevent internalized receptors from moving to lysosomes for degradation (20,21), Hap1 deficiency is likely to cause more degradation of internalized $\mathrm{TrkB}$ receptors and therefore impairment of $\mathrm{BDNF} /$ TrkB receptor-mediated signaling. Because JS is an autosomal recessive disorder, it would be interesting to examine whether loss of normal Ahi1 can also affect TrkB/BDNF signaling.

The potential significance of our findings is multifold. First, these studies suggest that BDNF-TrkB deficiency contributes to the pathogenesis of JS. Accordingly, the disease might be treated by addressing this deficiency. Second, our studies provide insight into cerebellar development. Since Hap1 and Ahi1 are selectively localized in distinct cerebellar nuclei, they can serve as markers for studying how these nuclei associate with other types of cerebellar neurons during the establishment of the cerebellar network. Third, it is hoped that the findings will help in the investigation of the normal Ahi1 function. We have provided evidence that Ahi1 and Hap1 interact tightly and that their interaction is necessary for maintaining their cellular levels. AHI1 seems to be involved not only in early brain development but also in the development of specific human leukemias and lymphomas $(12,13,46)$. The gene has also recently been reported as a candidate associated with susceptibility to schizophrenia $(47,48)$. Identification of its association with Hap1 will help elucidate its function in neurons and other types of cells.

\section{Methods}

Antibodies. Antibodies against Hap1 were generated in our previous studies $(16,22)$. GST fusion proteins containing N-terminal aa 83-174 of mouse Ahi1 were used as antigens for Covance to generate rabbit antibodies against Ahi1. The antiserum was affinity purified by incubation with a nitrocellulose strip containing transferred GST-Ahi1. Antibodies bound to the strip were then eluted with $0.2 \mathrm{mM}$ Tris-glycine, $\mathrm{pH} 2.8$, and neutralized by $1 \mathrm{M}$ Tris- $\mathrm{HCl}$, pH 8 . Characterization of Ahi1 antibodies involved the use of mouse brain tissues and transfected Ahi1 in HEK293 cells. Other antibodies used in the study were obtained from commercial sources as follows: rabbit anti-neurofilament (Chemicon; Millipore); mouse anti-tubulin (Sigma-Aldrich); rabbit anti-NeuN (Chemicon; Millipore); mouse antiTrkB (BD Biosciences); rabbit anti-calbindin (Sigma Aldrich), -Akt, -p-Akt, -p-Erk (Cell Signaling Technology), and -Erk (Santa Cruz Biotechnology Inc.); and mouse anti-biotin (Sigma-Aldrich).

Immunoprecipitation. Hap1-KO mice were generated in our previous study (19). All animal procedures were approved by the Institutional Animal Care and Use Committee of Emory University. Whole-brain extracts from 8 WT or Hap1-KO mouse pups at P1-P2 were combined and homogenized in HEPES buffer (20 mM HEPES, pH. 7.2, 1 mM DTT, 5 mM EDTA, and $100 \mu \mathrm{g} / \mathrm{ml} \mathrm{PMSF}$ ) containing protease inhibitor cocktail (Sigma-Aldrich). Homogenates were centrifuged at $160,000 \mathrm{~g}$ for 15 minutes, and supernatants were used for immunoprecipitation. Samples were adjusted to $2 \mathrm{ml}$ at $1.5 \mu \mathrm{g}$ protein $/ \mu \mathrm{l}$ and preabsorbed by protein A agarose beads $(100 \mu \mathrm{l}$; 1406-5G; Sigma-Aldrich) for 2 hours at $4^{\circ} \mathrm{C}$ with gentle rocking. Supernatants were collected and then incubated with $60 \mu \mathrm{l}$ of rabbit anti-Hap1 with gentle rocking overnight at $4{ }^{\circ} \mathrm{C}$ to isolate the Hap 1 protein complex. Next, $80 \mu \mathrm{l}$ of protein $\mathrm{A}$ beads were added for additional incubation at $4^{\circ} \mathrm{C}$ for 2 hours. Samples were spun in a tabletop microcentrifuge for $10-15 \mathrm{sec}-$ onds. Beads were extensively washed with lysis buffer containing Triton $\mathrm{X}-100$ (1\% final) and $\mathrm{NaCl}(0.6 \mathrm{M}$ final) and eluted with SDS sample buffer. The immunoprecipitated proteins were resolved by $8 \%$ SDS-PAGE and stained with Coomassie blue. Gel slices containing the protein of interest were excised and subjected to liquid chromatography-tandem mass spectrometry (LC-MS/MS) to reveal the identity of proteins. Immunoprecipitation of mouse brain lysates for 20 minutes was also performed to verify the results of the immunoisolation.

DNA constructs for expression of Abi1 and Hap1. cDNA plasmids encoding full-length mouse Ahi1 (aa 1-1,047) were generated in a previous study (13). We used PCR to generate truncated mouse Ahi1 (aa 1-284). These Ahil cDNAs were transferred to the PRK vector that links the influenza hemagglutinin (HA) epitope to the expressed Ahi1. PRK vectors containing Hap1A, Hap1B, or their GFP fusion proteins were generated in our previous studies (26). The cDNA constructs were transfected into human HEK293, rat PC12, and mouse N2A cells using Lipofectamine (Invitrogen).

GST fusion proteins containing full-length or aa 277-445 of rat Hap1 or full-length mouse Ahi1 were produced in bacterial strain BL21 (Amersham 
Biosciences) and purified with glutathione-agarose beads (Sigma-Aldrich) (16). For preabsorption assays to determine antibody specificity, rabbit anti-Ahi1 that had been incubated with GST-Ahi1 for 3 hours was used for Western blot analysis of mouse brain extract. HEK293 cells expressing transfected Ahi 1 were collected in buffer containing $1 \%$ Nonidet-P40, $10 \mathrm{mM}$ HEPES, $150 \mathrm{mM} \mathrm{NaCl}$, pH 7.4, and protease inhibitor mixture and sonicated for 30 seconds. Cell lysates were incubated with GST-Hap1 for the pull-down experiment using a previously described method (26).

Microscopy. Immunofluorescence staining of mouse brain sections was described in our previous study (22). Light micrographs were taken using a Zeiss microscope (Axiovert 200 MOT) equipped with a digital camera (ORCA-100; Hamamatsu) and the image acquisition software OpenLAB (Improvision). A $\times 20$ (LD-Achroplan $\times 20 / 0.4 \mathrm{NA})$ or $\times 63$ lens $(\times 63 / 0.75$ NA) was used for light microscopy. Confocal imaging was performed using the $\times 63 / 1.4$ NA oil immersion objective lens (Plan-Apochromat) and a Zeiss LSM 510 confocal microscope system (Zeiss). Enhanced GFP was imaged using 488-nm excitation and a 500- to 530-nm band-pass filter, and red fluorescent protein was imaged using $543-\mathrm{nm}$ excitation and a 565- to 615-nm band-pass filter. The figures were created using Photoshop 7.0 software (Adobe).

Neuronal cell culture. For culturing rat brainstem cells, we isolated cells from the brainstem of embryonic rats at E17-E18. For culturing brainstem cells from Hap1-KO mice, we isolated them from mouse brains at P1 using a previously described method (49). Cerebellar neurons were isolated from mice at P3-P5. These neurons were cultured in the medium of Neurobasal A containing B27. After 4-5 days, the cells were treated with BDNF or adenoviral siRNA. Adenoviral siRNA for Ahi1 was generated by Welgen using the siRNA sequence 1762-GCCACCTCAATATCATTTA-1780 under the control of the $\mathrm{U} 6$ promoter in a vector that also independently expresses GFP by the CMV promoter. Adenoviral siRNA for Hap1 was generated using the same vector and was described previously (32). Control virus that expresses GFP alone served as a control. The viral titer was determined by measuring the number of infected HEK293 cells expressing GFP.

Biotinylation of BDNF assay. Biotinylation of membrane and internalized proteins was performed using a previously described method (22). Cultured brainstem cells were incubated with NHS-SS-biotin $(1.0 \mathrm{mg} / \mathrm{ml}$ in PBS-Ca-Mg) for 30 minutes at $4^{\circ} \mathrm{C}$. Unbound BDNF-biotin was washed off with culture medium. Internalization was initiated by incubating the cultures with warm media $\left(37^{\circ} \mathrm{C}\right)$ for 30 minutes at $37^{\circ} \mathrm{C}$. The cultures were then washed with ice-cold acid for 20 minutes to remove the surfacebound BDNF, fixed in 4\% paraformaldehyde in PBS, and permeabilized with $0.2 \%$ Triton $\mathrm{X}-100$. The internalized BDNF-biotin was visualized by anti-biotin antibody $(1: 10,000)$ and immunofluorescence staining.

To measure internalized membrane TrkB via Western blotting, serumstarved brainstem neurons were subjected to biotinylation as described above. To prevent lysosomal degradation of internalized TrkB, cells were treated with $100 \mu \mathrm{g} / \mathrm{ml}$ of the lysosomal protease inhibitor leupeptin for 30 minutes before biotinylation. The biotin remaining at the cell surface was removed with cleaving buffer $(50 \mathrm{mM}$ glutathione, $75 \mathrm{~mm} \mathrm{NaCl}, 10 \mathrm{mM}$ EDTA, $1 \%$ BSA, and $0.075 \mathrm{~N} \mathrm{NaOH}$ ) before precipitation. The membrane and cytosolic fractions were prepared and lysed with immunoprecipitation assay buffer (50 mm Tris- $\mathrm{HCl}$, pH 8.0, $150 \mathrm{mM} \mathrm{NaCl}, 1 \mathrm{~mm}$ EDTA, $1 \mathrm{mM}$ EGTA, $0.1 \%$ SDS, $0.5 \%$ deoxycholate, and $1 \%$ Triton X-100). Biotinylated proteins were precipitated by $40 \mu \mathrm{l}$ (1:1 slurry) of UltraLink NeutrAvidin beads (Pierce), resuspended in $80 \mu \mathrm{l}$ of SDS-PAGE sample buffer, and analyzed by Western blotting with anti-TrkB. Supernatant after immunoprecipitation was also probed with different antibodies.

Statistics. All values are expressed as mean \pm SEM. Statistical significance was assessed by the use of 2-tailed Student's $t$ test, and a $P$ value of less than 0.05 was considered significant.

\section{Acknowledgments}

We thank Zhihui Fang and Adam Orr for technical assistance and Meyer Friedman and Cheryl Strauss for critical reading of the manuscript. The work was supported by NIH grants NS36232, AG19206 (to X.-J. Li), NS045016 (to S.-H. Li), and P30NS055077 (to J. Peng).

Received for publication February 14, 2008, and accepted in revised form May 28, 2008.

Address correspondence to: Xiao-Jiang Li, Department of Human Genetics, Emory University School of Medicine, 615 Michael St., Atlanta, Georgia 30322, USA. Phone: (404) 727-3290; Fax: (404) 727-3949; E-mail: xiaoli@genetics.emory.edu.
1. Parisi, M.A., Doherty, D., Chance, P.F., and Glass, I.A. 2007. Joubert syndrome (and related disorders) (OMIM 213300). Eur. J. Hum. Genet. 15:511-521.

2. Maria, B.L., et al. 1999. Molar tooth sign in Joubert syndrome: clinical, radiologic, and pathologic significance. J. Child Neurol. 14:368-376.

3. Louie, C.M., and Gleeson, J.G. 2005. Genetic basis of Joubert syndrome and related disorders of cerebellar development. Hum. Mol. Genet. 14:R235-R242.

4. Saar, K., et al. 1999. Homozygosity mapping in families with Joubert syndrome identifies a locus on chromosome 9q34.3 and evidence for genetic heterogeneity. Am. J. Hum. Genet. 65:1666-1671.

5. Keeler, L.C., et al. 2003. Linkage analysis in families with Joubert syndrome plus oculo-renal involvement identifies the CORS2 locus on chromosome 11p12-q13.3. Am. J. Hum. Genet. 73:656-662.

6. Lagier-Tourenne, C., et al. 2004. Homozygosity mapping of a third Joubert syndrome locus to 6q23. J. Med. Genet. 41:273-277.

7. Pellegrino, J.E., Lensch, M.W., Muenke, M., and Chance, P.F. 1997. Clinical and molecular analysis in Joubert syndrome. Am J. Med. Genet. 72:59-62.

8. Blair, I.P., Gibson, R.R., Bennett, C.L., and Chance, P.F. 2002. Search for genes involved in Joubert syndrome: evidence that one or more major loci are yet to be identified and exclusion of candidate genes EN1, EN2, FGF8, and BARHL1. Am. J. Med. Genet. 107:190-196.
9. Bennett, C.L., et al. 2004. Joubert syndrome: a haplotype segregation strategy and exclusion of the zinc finger protein of cerebellum 1 (ZIC1) gene. Am. J. Med. Genet. A. 125A:117-124; discussion 117.

10. Ferland, R.J., et al. 2004. Abnormal cerebellar development and axonal decussation due to mutations in AHI1 in Joubert syndrome. Nat. Genet. 36:1008-1013.

11. Dixon-Salazar, T., et al. 2004. Mutations in the AHI1 gene, encoding jouberin, cause Joubert syndrome with cortical polymicrogyria. Am. J. Hum. Genet. 75:979-987.

12. Poirier, Y., and Jolicoeur, P. 1989. Distinct helper virus requirements for Abelson murine leukemia virus-induced pre-B- and T-cell lymphomas. J. Virol. 63:2088-2098

13. Jiang, X., Hanna, Z., Kaouass, M., Girard, L., and Jolicoeur, P. 2002. Ahi-1, a novel gene encoding a modular protein with WD40-repeat and SH3 domains, is targeted by the Ahi- 1 and Mis- 2 provirus integrations. J. Virol. 76:9046-9059.

14. Smith, T.F., Gaitatzes, C., Saxena, K., and Neer, E.J. 1999. The WD repeat: a common architecture for diverse functions. Trends Biochem. Sci. 24:181-185.

15. Mayer, B.J. 2001. SH3 domains: complexity in moderation. J. Cell Sci. 114:1253-1263.

16. Li, X.J., et al. 1995. A huntingtin-associated protein enriched in brain with implications for pathology. Nature. 378:398-402.
17. Gutekunst, C.A., et al. 1998. The cellular and subcellular localization of huntingtin-associated protein 1 (HAP1): comparison with huntingtin in rat and human. J. Neurosci. 18:7674-7686.

18. Chan, E.Y., et al. 2002. Targeted disruption of Huntingtin-associated protein-1 (Hap1) results in postnatal death due to depressed feeding behavior. Hum. Mol. Genet. 11:945-959.

19. Li, S.H., et al. 2003. Lack of huntingtin-associated protein-1 causes neuronal death resembling hypothalamic degeneration in Huntington's disease. J. Neurosci. 23:6956-6964.

20. Li, Y., Chin, L.S., Levey, A.I., and Li, L. 2002. Huntingtin-associated protein 1 interacts with hepatocyte growth factor-regulated tyrosine kinase substrate and functions in endosomal trafficking. J. Biol. Chem. 277:28212-28221.

21. Kittler, J.T., et al. 2004. Huntingtin-associated protein 1 regulates inhibitory synaptic transmission by modulating gamma-aminobutyric acid type A receptor membrane trafficking. Proc. Natl. Acad. Sci. U. S. A. 101:12736-12741.

22. Rong, J., et al. 2006. Regulation of intracellular trafficking of huntingtin-associated protein-1 is critical for TrkA protein levels and neurite outgrowth. J. Neurosci. 26:6019-6030.

23. Gauthier, L.R., et al. 2004. Huntingtin controls neurotrophic support and survival of neurons by enhancing BDNF vesicular transport along micro- 
tubules. Cell. 118:127-138.

24. Page, K.J., Potter, L., Aronni, S., Everitt, B.J., and Dunnett, S.B. 1998. The expression of Huntingtinassociated protein (HAP1) mRNA in developing, adult and ageing rat CNS: implications for Huntington's disease neuropathology. Eur. J. Neurosci. 10:1835-1845.

25. Millen, K.J., Wurst, W., Herrup, K., and Joyner, A.L. 1994. Abnormal embryonic cerebellar development and patterning of postnatal foliation in two mouse Engrailed-2 mutants. Development. 120:695-706.

26. McGuire, J.R., Rong, J., Li, S.H., and Li, X.J. 2006. Interaction of huntingtin-associated protein- 1 with kinesin light chain: implications in intracellular trafficking in neurons. J. Biol. Chem. 281:3552-3559.

27. Aruga, J., et al. 1998. Mouse Zic1 is involved in cerebellar development. J. Neurosci. 18:284-293.

28. Barbacid, M. 1994. The Trk family of neurotrophin receptors. J. Neurobiol. 25:1386-1403.

29. Chao, M.V. 2003. Neurotrophins and their receptors: a convergence point for many signalling pathways. Nat. Rev. Neurosci. 4:299-309.

30. Schwartz, P.M., et al. 1997. Abnormal cerebellar development and foliation in $\mathrm{BDNF}^{-/}$- mice reveals a role for neurotrophins in CNS patterning. Newron. 19:269-281.

31. Borghesani, P.R., et al. 2002. BDNF stimulates migration of cerebellar granule cells. Development. 129:1435-1442.

32. Sheng, G., et al. 2006. Hypothalamic huntingtin-associated protein 1 as a mediator of feeding behavior. Nat. Med. 12:526-533.

33. Du, J., et al. 2003. Regulation of TrkB receptor tyrosine kinase and its internalization by neuronal activity and Ca2+ influx. J. Cell Biol. 163:385-395.

34. Li, S.H., Gutekunst, C.A., Hersch, S.M., and Li, X.J. 1998. Interaction of huntingtin-associated protein with dynactin P150Glued. J. Neurosci. 18:1261-1269.

35. Li, X.J., and Li, S.H. 2005. HAP1 and intracellular trafficking. Trends Pharmacol. Sci. 26:1-3.

36. Shinoda, K., Nagano, M., and Osawa, Y. 1993. An aromatase-associated cytoplasmic inclusion, the "stigmoid body," in the rat brain: II. Ultrastructure (with a review of its history and nomenclature). J. Comp. Neurol. 329:1-19.

37. Hatten, M.E., and Heintz, N. 1995. Mechanisms of neural patterning and specification in the developing cerebellum. Annu. Rev. Neurosci. 18:385-408.

38. Baader, S.L., Sanlioglu, S., Berrebi, A.S., ParkerThornburg, J., and Oberdick, J. 1998. Ectopic overexpression of engrailed-2 in cerebellar Purkinje cells causes restricted cell loss and retarded external germinal layer development at lobule junctions. J. Neurosci. 18:1763-1773.

39. Voogd, J., and Glickstein, M. 1998. The anatomy of the cerebellum. Trends Neurosci. 21:370-375.

40. Wang, V.Y., and Zoghbi, H.Y. 2001. Genetic regulation of cerebellar development. Nat. Rev. Neurosci. 2:484-491.

41. Liao, M., et al. 2005. Immunohistochemical localization of huntingtin-associated protein 1 in endocrine system of the rat. J. Histochem. Cytochem.
53:1517-1524.

42. Ye, H., Kuruvilla, R., Zweifel, L.S., and Ginty, D.D. 2003. Evidence in support of signaling endosomebased retrograde survival of sympathetic neurons. Neuron. 39:57-68.

43. Rico, B., Xu, B., and Reichardt, L.F. 2002. TrkB receptor signaling is required for establishment of GABAergic synapses in the cerebellum. Nat. Neurosci. 5:225-233.

44. Klein, R., et al. 1993. Targeted disruption of the trkB neurotrophin receptor gene results in nervous system lesions and neonatal death. Cell. 75:113-122.

45. Minichiello, L., and Klein, R. 1996. TrkB and TrkC neurotrophin receptors cooperate in promoting survival of hippocampal and cerebellar granule neurons. Genes Dev. 10:2849-2858.

46. Ringrose, A., et al. 2006. Evidence for an oncogenic role of AHI-1 in Sezary syndrome, a leukemic variant of human cutaneous T-cell lymphomas. Leukemia. 20:1593-1601.

47. Amann-Zalcenstein, D., et al. 2006. AHI1, a pivotal neurodevelopmental gene, and C6orf217 are associated with susceptibility to schizophrenia. Eur.J. Hum. Genet. 14:1111-1119.

48. Ingason, A., et al. 2007. Support for involvement of the AHI1 locus in schizophrenia. Eur. J. Hum. Genet. 15:988-991.

49. Kivell, B.M., McDonald, F.J., and Miller, J.H. 2001. Method for serum-free culture of late fetal and early postnatal rat brainstem neurons. Brain Res. Brain Res. Protoc. 6:91-99. 